\title{
ACCURACY OF ULTRASOUND IN DETECTION OF HEPATOCELLULAR CARCINOMA IN CIRRHOTIC PATIENTS.
}

1. MBBS FCPS (Radiology) Consultant Radiologist DHQ Hospital Faisalabad.

2. MBBS FCPS Radiology Consultant Radiologist DHQ Hospital Faisalabad.

3. MBBS, MS, FCPS, FANMB (Japan) Consultant Radiologist \& Nuclear Physician PMO, PINUM

4. MBBS, FCPS (Radiology) Consultant Radiogist

Allied Hospital/Faisalabad Medical University, Faisalabad.

5. FCPS

Allied Hospital, Faisalabad

6. MBBS

PG Trainee

Department of Radiology and Medical Imaging

Allied Hospital/Faisalabad Medical University, Faisalabad.

\section{Correspondence Address:}

Dr. Bushra Riaz

P-737 Street 1 Dawood Colony

Ali Town Sargodha Road Faisalabad. bushrariaz15@hotmail.com

Article received on: 02/08/2018

Accepted for publication: 03/12/2019

\begin{abstract}
Bushra Riaz', Rakhshanda Jabbar², Owais Bin Qadeer³, Nasira Parveen, Fouzia Sultan ${ }^{5}$,
\end{abstract} Shakeel Ahmad ${ }^{6}$

ABSTRACT... Objectives: Evaluate the accuracy of ultrasonography in the diagnosis of hepatocellular carcinoma among cirrhotic patients by comparing it with contrast enhanced multiphasic MRI. Study Design: Cross sectional study. Setting: Radiology Department through OPD $\backslash$ Admittedlemergency, Allied Hospital, Faisalabad. Period: 01-01-2018 to 3006-2018. Material \& Methods: A total of 91 cases, having symptoms of fatigue, weakness, vomiting, nausea, loss of appetite, abdominal pain, weight loss and bloating when the fluid is accumulated in abdominal cavity, itching, spider-like blood vessels on the skin were included in the study. Informed written consent was taken from all the patients or their next of kin after explaining the procedure, objectives and benefits of study. All the patients were examined with Conventional gray scale ultrasound using curvilinear probe of $5 \mathrm{MHz}$ and then with 1.5 tesla MR imager. Results: The diagnostic accuracy was computed as $42.86 \%(n=39)$ as true positive, $1.10 \%(n=1)$ as false positive, $49.45 \%(n=45)$ false negative and $6.59 \%(n=6)$ had true negative, while $46.43 \%$ (sensitivity), $85.71 \%$ (specificity), 97.5\%(positive predictive value), 88.24\%(negative predictive value), and $49.45 \%$ (diagnostic accuracy) were calculated. Conclusion: We concluded that the accuracy of ultrasonography for the diagnosis of hepatocellular carcinoma among cirrhotic patients by comparing it with contrast enhanced multiphasic MRI is in agreement with the previous studies, though its accuracy rate is lower but specificity is significantly higher and recommended in our country in low resource settings and tertiary care setups where diagnostic facilities are limited functional.

Key words: Cirrhotic Patients, Diagnostic Accuracy, Hepatocellular Carcinoma, Ultrasonography.

Article Citation: Riaz B, Jabbar R, Qadeer OB, Parveen N, Sultan F, Ahmad S. Accuracy of ultrasound in detection of Hepatocellular Carcinoma in cirrhotic patients. Professional Med J 2020; 27(1):197-200.

DOI: 10.29309/TPMJ/2020.27.1.4404

\section{INTRODUCTION}

Primary liver malignancies are recorded with a dramatic increase in last 20 years, similar to hepatocellular carcinoma (HCC) being a common liver tumor worldwide. Now, HCC is considered as the commonest cause of cancer death all over the world. ${ }^{1}$ Early and appropriate management of this morbidity is based on early detection. Hepatobiliary MRI is known as a useful technique while evaluating regenerating and dysplactic nodules. Various authors ${ }^{2-3}$ are of the view that $M R I$ is better than $C T$ for detection and characterization of focal lesions. However, MRI has emerged as a useful diagnostic modality for evaluation of cirrhosis and its associated complications. Faster sequences and automated contrast detection allow reproducible capture of arterial phase, being essential for detection and characterization of $\mathrm{HCC}^{4}$

Multiphasic dynamic gadolinium contrast enhanced T1 W in hepatic arterial, portal venous and delayed phases improve the detection and characterization of lesions, particularly small $\mathrm{HCC}$, and they are superior to multiphasic helical

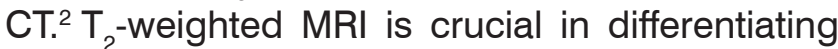
HCC'S from pre-malignant or borderline lesions in cirrhosis and serves as a tie breaker for small early-enhancing lesions. Gadolinium-enhanced MRI has advantages over contrast-enhanced CT: greater contrast enhancement, smaller volume of contrast, and less frequent adverse reactions. Double hepatic arterial-phase imaging has been performed in may center, allowing 
less frequent off-timing arterial-phase imaging and improved temporary resolution. ${ }^{3}$ In addition, use of tissue-specific contrast agents such as super paramagnetic iron oxide, allows improved detection and characterization of liver tumours. 5,6 Frequency of $\mathrm{HCC}$ in cirrhotic patients using contrast enhance multiphasic MRI is $96 \% .^{7-8}$ Frequency of HCC detection using US has been reported to be between $38 \%-63.9 \% .^{9}$

The rationale of this study to utilize the contrast enhanced multiphasic MRI as an early diagnostic tool for HCC in patients with cirrhosis of liver, so that different treatment options like resection, transplantation, tumor ablation and chemoembolization can be offered to the patients at the early stage of disease. This study is thus being carried out to determine the accuracy of US in $\mathrm{HCC}$. If this is found to be accurate then this may be used in low resource settings as well as in many tertiary care setups where there are limited functional diagnostic facilities.

\section{METHODOLOGY}

The study protocol was been approved by hospital ethical committee. Cirrhotic patients presenting in Radiology department through OPD\Admitted \ emergency having symptoms of fatigue, weakness, vomiting, nausea, loss of appetite, abdominal pain, weight loss and bloating when the fluid is accumulated in abdominal cavity, itching, spider-like blood vessels on the skin was included in the study. Informed written consent was taken from all the patients or their next of kin after explaining the procedure, objectives and benefits of study.

All the patients were examined with Conventional gray scale ultrasound using curvilinear probe of $5 \mathrm{MHz}$ and then with 1.5 tesla MR imager. Multiphasic contrast enhance MRI liver was included breadth hold Turbo spin echo sequences acquired before the contrast injection as well as post contrast sequences in the arterial phase, portal venous phase and delayed phase. Sense body coil was used for MRI examination. MRI protocol included T1 Weighted, T2 Weighted and fat suppression images in transverse and coronal plane having $5 \mathrm{~mm}$ slice thickness, slice gap of
$1 \mathrm{~mm}$, flip angle 75 degrees, reconstruction matrix of 512 , voxel size $0.74 \mathrm{~mm}$, element selection 1234 and TR and TE were taken shortest in T1 Weighted images and fat suppression images and longest in T2 Weighted images. The conventional USG and Multiphasic MRI examination of liver was performed in the radiology department and I interpreted it for reporting under supervision of consultant radiologist. Frequency and \% were calculated for gender and hepatocellular carcinoma on US \& multiphasic MRI \& True positives. All the data was presented in the form of tables.

\section{RESULTS}

Age distribution of the patients was done which shows that $23.08 \%(n=21)$ were between $15-30$ years, $41.76 \%(n=38)$ between $31-60$ years and $35.16 \%(n=32)$ were between $61-80$ years of age, mean \pm sd was calculated as $46.98 \pm 5.62$ years. Gender distribution of the patients was done which shows that $70.33 \%(n=64)$ were male and $29.67 \%(n=27)$ were females.

Frequency of hepatocellular carcinoma on multiphasic MRI reveals $92.31 \%(n=84)$ patients with positive hepatocellular carcinoma while $7.69 \%(n=7)$ has no findings of HCC. (Table No. I)

\begin{tabular}{|c|c|c|}
\hline Age(in years) & No. of patients & $\%$ \\
\hline $15-30$ & 21 & 23.08 \\
\hline $31-60$ & 38 & 41.76 \\
\hline $61-80$ & 32 & 35.16 \\
\hline Total & 91 & 100 \\
\hline
\end{tabular}

Gender distribution of the patients was done which shows that $70.33 \%(n=64)$ were male and $29.67 \%(n=27)$ were females. (Table No. II)

\begin{tabular}{|c|c|c|}
\hline Gender & No. of patients & $\%$ \\
\hline Male & 64 & 70.33 \\
\hline Female & 27 & 29.67 \\
\hline Total & $\mathbf{9 1}$ & $\mathbf{1 0 0}$ \\
\hline Table-II. Gender distribution $(\mathbf{n}=\mathbf{9 1})$ \\
\hline
\end{tabular}


In Table III, diagnostic accuracy was computed as $42.86 \%(n=39)$ as true positive, $1.10 \%(n=1)$ as false positive, $49.45 \%(n=45)$ false negative and $6.59 \%(n=6)$ had true negative, while 46.43\%(sensitivity), $85.71 \%$ (specificity), $97.5 \%$ (positive predictive value), $88.24 \%$ (negative predictive value), and 49.45\%(diagnostic accuracy) were calculated.

\begin{tabular}{|c|c|c|c|}
\hline \multirow{2}{*}{ Ultrasound } & \multicolumn{2}{|c|}{ MRI } & \multirow{2}{*}{ Total } \\
\hline & Positive & Negative & \\
\hline Positive & $\begin{array}{c}\text { True } \\
\text { positive(a) } \\
39(42.86 \%)\end{array}$ & $\begin{array}{c}\text { False } \\
\text { positive (b) } \\
1(1.10 \%)\end{array}$ & $\begin{array}{c}a+b \\
40(43.96 \%)\end{array}$ \\
\hline Negative & $\begin{array}{c}\text { False } \\
\text { negative(c) } \\
45(49.45 \%)\end{array}$ & $\begin{array}{c}\text { True } \\
\text { negative } \\
(\mathrm{d}) \\
6(6.59 \%)\end{array}$ & $\begin{array}{c}C+d \\
51(56.04 \%)\end{array}$ \\
\hline Total & $\begin{array}{c}a+c \\
84(92.31 \%)\end{array}$ & $\begin{array}{c}b+d \\
7(7.69 \%)\end{array}$ & $91(100 \%)$ \\
\hline
\end{tabular}

Table-III. Diagnostic accuracy of ultrasonography to detect hepatocellular carcinoma among cirrhotic patients $(n=91)$

Sensitivity $\quad=a /(a+c) \times 100=46.43 \%$

Specificity $\quad=d /(d+b) \times 100=85.71 \%$

Positive predictive value $=a /(a+b) \times 100=97.5 \%$

Negative predictive value $=d /(d+c) \times 100=88.24 \%$

Accuracy rate $=a+d /(a+d+b+c) \times 100=49.45 \%$

\section{DISCUSSION}

We planned this study with the view to determine the accuracy of US in HCC. If this is found to be accurate then this may be used in low resource settings as well as in many tertiary care setups where there are limited functional diagnostic facilities.

In our study, $23.08 \%(n=21)$ were between $15-30$ years, $41.76 \%(n=38)$ between $31-60$ years and $35.16 \%(n=32)$ were between $61-80$ years of age, mean + sd was calculated as $46.98 \pm 5.62$ years, $70.33 \%(n=64)$ were male and $29.67 \%(n=27)$ were females, frequency of hepatocellular carcinoma on multiphasic $\mathrm{MRI}$ reveals $92.31 \%(n=84)$ patients with positive hepatocellular carcinoma while $7.69 \%(n=7)$ has no findings of HCC while diagnostic accuracy of ultrasonography to detect hepatocellular carcinoma among cirrhotic patients was computed as $42.86 \%(n=39)$ true positive, $1.10 \%(n=1)$ as false positive,
$49.45 \%(n=45)$ false negative and $6.59 \%(n=6)$ had true negative, while $46.43 \%$ (sensitivity), $85.71 \%$ (specificity), $\quad 97.5 \%$ (positive predictive value), $88.24 \%$ (negative predictive value), and $49.45 \%$ (diagnostic accuracy) were calculated.

The other studies also confirmed that using USG for detection of HCC is in practiced with acceptable level of sensitivity i.e. $65-80 \%$ while the specificity is $>90 \% .^{10-12}$ The size of tumor is of significant effect while detecting HCC on US. The sensitivity ranges for $42 \%$ for the lesions $\leq 1 \mathrm{~cm}^{13-14}$ to $95 \%$ for larger sized tumors. ${ }^{15}$ The sensitivity is poor when used for pre-transplant screening due to echotexture coarse of liver and the frequent presence of higher malignant lesions and frequent ascites in cases with end staged liver disease. ${ }^{16} \mathrm{~A}$ previous retrospective trial consisting of 200 cases undergoing liver transplantation within 12 weeks of last screening, the US scanning was recorded to be correlated with explanted livers, while the sensitivity was ranged from $13.5 \%$ to $50 \%$ of lesions between 1-5 cm of diameter. ${ }^{17}$ However, CT/MRI should be used in cases with liver transplant. ${ }^{18}$

In our study being the limitation of the study we did not differentiated the sensitivity according to the size of the lesion, however, it is recommended that in further trials the differentiation may be done to further authenticate the results of the study, on the hand, this technique may be used in low resource settings as well as in many tertiary care setups where there are limited functional diagnostic facilities as we recorded a higher specificity.

\section{CONCLUSION}

Weconcluded thattheaccuracy ofultrasonography for the diagnosis of hepatocellular carcinoma among cirrhotic patients by comparing it with contrast enhanced multiphasic MRI is in agreement with the previous studies, though its accuracy rate is lower but specificity is significantly higher and recommended in our country in low resource settings and tertiary care setups where diagnostic facilities are limited functional.

Copyright@ 03 Dec, 2019. 


\section{REFERENCES}

1. Ariff $B$, Lloyd $C R$, Khan $S$, Shariff M, Thillainayagam AV, Bansi DS et al. Imaging of liver cancer. World $J$ Gastroenterol 2009;15:1289-1300.

2. Digumarthy SR, Sahani DV, Saini S. MRI in detection of hepatocellular carcinoma (HCC). Cancer Imaging. 2005;5:20-4.

3. Kanematsu M, Kondo H, Goshima S, Tsuge Y, Watanabe $\mathrm{H}$. Magnetic Resonance Imaging of Hepatocellular Carcinoma. Oncology 2008;75:65-71.

4. Hussain SM, Reinhold C, Mitchell DG. Cirrhosis and lesion characterization at MR imaging. Radiographics 2009;29:1637-52.

5. Bellin MF. MR contrast agents, the old and the new. Eur J Radiol. 2006;60:314-323.

6. Gandhi SN, Brown MA, Wong JG, Aguirre DA, Sirlin CB. MR contrast agents for liver imaging: what, when, how. Radiographics. 2006;26:1621-36.

7. Hanna RF, Kased N, Kwan SW, Gamst AC, Santosa AC. Double-contrast MRI for accurate staging of hepatocellular cancinoma in patients with cirrhosis; AJR 2008;190:47-57.

8. Singh P. EUS for detection of the hepatocellular carcinoma: results of a prospective study. Gastrointest Endosc 2007;66(2):265-73.

9. Tanaka S, Kitamura T. Recent advances in ultrasonographic diagnosis of hepatocellular carcinoma. Cancer. 1989;63(7):1313-7.

10. Bolondi L, Sofia S, Siringo S, Gaiani S, Casali A, Zironi G, Piscaglia F, Gramantieri L, Zanetti M, Sherman M. Surveillance programme of cirrhotic patients for early diagnosis and treatment of hepatocellular carcinoma: a cost effectiveness analysis. Gut 2001;48:251-9.
11. Bruix J, Sherman M. Management of hepatocellular carcinoma. Hepatology. 2005;42:1208-36.

12. Bruix J, Hessheimer AJ, Forner A, Boix L, Vilana R, Llovet JM. New aspects of diagnosis and therapy of hepatocellular carcinoma. Oncogene. 2006;25:384856.

13. Dodd GD 3rd, Miller WJ, Baron RL, Skolnick ML, Campbell WL. Detection of malignant tumors in end-stage cirrhotic livers: efficacy of sonography as a screening technique. AJR Am J Roentgenol. 1992;159:727-33.

14. Franca AV, Elias Junior J, Lima BL, Martinelli AL, Carrilho FJ. Diagnosis, staging and treatment of hepatocellular carcinoma. Braz J Med Biol Res. 2004;37:1689-1705.

15. Colli A, Fraquelli M, Casazza G, Massironi S, Colucci A, Conte D, Duca P. Accuracy of ultrasonography, spiral $\mathrm{CT}$, magnetic resonance, and alpha-fetoprotein in diagnosing hepatocellular carcinoma: a systematic review. Am J Gastroenterol. 2006;101:513-23.

16. Saar B, Kellner-Weldon F. Radiological diagnosis of hepatocellular carcinoma. Liver Int. 2008;28:189-99.

17. Bennett GL, Krinsky GA, Abitbol RJ, Kim SY, Theise ND, Teperman LW. Sonographic detection of hepatocellular carcinoma and dysplastic nodules in cirrhosis: correlation of pretransplantation sonography and liver explant pathology in 200 patients. AJR Am J Roentgenol. 2002;179:75-80.

18. Saab S, Ly D, Nieto J, Kanwal F, Lu D, Raman S, et al. Hepatocellular carcinoma screening in patients waiting for liver transplantation: a decision analytic model. Liver Transpl. 2003;9:672-81.

\begin{tabular}{|c|l|l|}
\hline \multicolumn{3}{|c}{ AUTHORSHIP AND CONTRIBUTION DECLARATION } \\
\hline Sr. \# & \multicolumn{1}{|c|}{ Author(s) Full Name } & \multicolumn{1}{|c|}{ Contribution to the paper } \\
\hline 1 & Bushra Riaz & Main author, Data collection. \\
2 & Rakhshanda Jabbar & Literature review. \\
\hline 3 & Owais Bin Qadeer & Collection of References. \\
\hline 4 & Nasira Parveen & Literature review. \\
\hline 5 & Fouzia Sultan & Literature review. \\
6 & Shakeel Ahmad & Literature review. \\
\hline
\end{tabular}

